\title{
Coordinating Handover Parameter Optimization and Load Balancing in LTE Self-Optimizing Networks
}

\author{
Andreas Lobinger*, Szymon Stefanski ${ }^{\dagger}$, Thomas Jansen ${ }^{\ddagger}$, Irina Balan ${ }^{\S}$ \\ * Nokia Siemens Networks, München, Germany, andreas.lobinger@nsn.com \\ $\dagger$ Nokia Siemens Networks, Wrocław, Poland, szymon.stefanski@nsn.com \\ $\ddagger$ Technical University of Braunschweig, Braunschweig, Germany, Jansen@ifn.ing.tu-bs.de \\ §Interdisciplinary Institute for Broadband Technology, Ghent, Belgium, Irina.Balan@intec.ugent.be
}

\begin{abstract}
In this paper we present simulation results of a self-optimizing network in a long-term-evolution (LTE) mobile communication system that uses two optimizing algorithms at the same time: load balancing (LB) and handover parameter optimization (HPO). Based on previous work [1][2][5], we extend the optimization by a combined use case. We present the interactions of the two SON algorithms and show an example of a coordination system. The coordination system for self optimization observes system performance and controls the SON algorithms. As both SON algorithms deal with the handover decision itself, not only interactions, but also conflicts in the observation and control of the system are to be expected and are observed. The example of a coordination system here is not the optimal solution covering all aspects, but rather a working solution that shows equal performance to the individual algorithms or in the best case combining the strengths of the algorithms and achieving even better performance; although as localized gain, in time and area.
\end{abstract}

\section{INTRODUCTION}

In existing networks, parameters are manually adjusted to obtain a high level of network operational performance. In LTE the concept of self-optimizing networks (SON) is introduced, where the parameter tuning is done automatically based on measurements. A challenge is to deliver additional performance gain further improving network efficiency. Two individual SON algorithms are presented here: load balancing as means to offload excess traffic to low-loaded adjacent cells and handover parameter optimization as means to reduce handover (HO) problems like radio link failures, handover failures or ping-pong handovers. Simulations were conducted for a synthetic, non regular network layout and the simulation models deal with the radio channels and radio connections in a standard LTE downlink system [4].

The work has been carried out in the EU FP7 SOCRATES project [1].

\section{HANDOVER PROCEDURE AND PARAMETERS}

The main procedure to provide mobility management in cellular mobile communication systems is the handover procedure. In the case of LTE networks, the procedure starts with the measurement reporting (MR) sent by the user equipment (UE) to the serving eNB (SeNB). The UE periodically performs downlink radio channel measurements of the reference symbols received power (RSRP) on the pilot channel. If certain network configured conditions are satisfied, the UE sends the

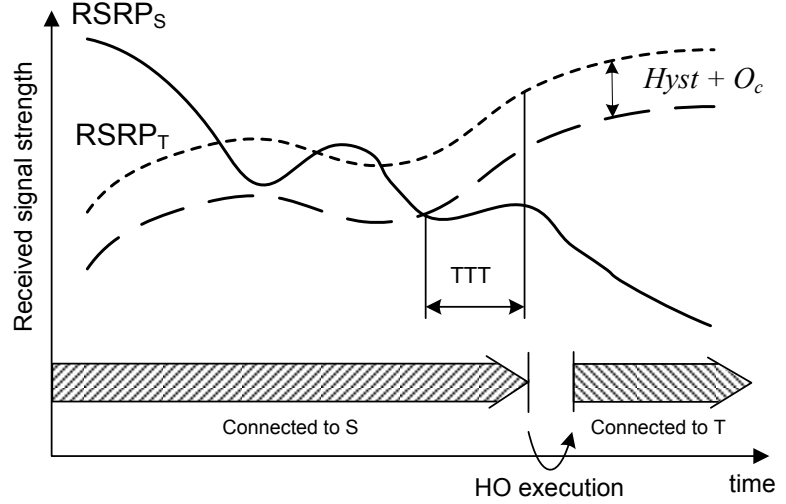

Fig. 1. Handover procedure as timeline of RSRP values for two cells, indicating the TTT and the change of connection

corresponding MR indicating the triggered event. In our case, the measurement report trigger is condition A3 [8], a relative condition between cells. The decision to handover a UE from serving cell $\mathrm{S}$ to target cell $\mathrm{T}$ is taken if the condition in equation 1 is true for a certain amount of time. In addition, based on the MR, the cell indicates to which cell the UE shall be handed over to, namely target eNB (TeNB).

$$
R S R P_{S}+O_{c}>R S R P_{T}+\text { Hyst }
$$

with $R S R P$ as the signal strength values for serving $S$ and target $T$ cell, $O_{c}$ as specific offset and Hyst as cell specific Hysteresis value. Figure 1 shows a timeline of RSRP values of two cells, together with additional offsets and hysteresis, a measurement trigger taking place and the timeline of the connection. The hysteresis Hyst -which is a cell specific value - is a means to ensure the decision for a significantly better cell (Hyst higher than the current serving cell). The Time-to-Trigger TTT delays and ensures the decision for the better cell also, by forcing the condition to hold true for as long as TTT. The offset $O_{c}$ is a cell pair specific value, which shifts the actual cell border, i.e. the point at which pathloss to both eNB is equal, to one or the other cells direction.

The SeNB will subsequently communicate with that target cell and the UE will be controlled to close the radio link to its serving cell and start a new radio connection in the target cell. 


\section{LOAD BALANCING AND HANDOVER PARAMETERS OPTIMIZATION ALGORITHMS}

The key idea of load balancing [2] is to shift the load of overloaded cells to less loaded adjacent cells by changing the virtual cell borders. The respective UEs are handed over with a load balancing handover command after the virtual cell borders have been changed. Hence it is averted that the UEs will be handed back immediately. The change is done by controlling the cell pair specific offsets $O_{c}$ coordinated on cell pair basis [6]. The decisions of the algorithm are based on a virtual load measure that can exceed $100 \%$ load level and thus detect an overload. The virtual load can be seen as relation of the sum of resources needed to achieve a certain QoS for all active UEs (related to the traffic and service model) divided by the number of resources available to the scheduler. In this context we define an Unsatisfied Users Metric that is derived from the virtual load measure and accounts the number of unsatisfied users i.e. users that do not achieve the QoS for the defined traffic and service model. In these simulations a constant bitrate traffic (CBR) of $256 \mathrm{kBit} / \mathrm{s}$ and a Quality of Service (QoS) of $100 \%$ of that rate have been defined. In [3] a mathematical framework for SON investigations on the downlink is defined and in [2] a detailed description of the used load balancing algorithm is provided.

The key idea for the HPO algorithm [5] is to steer the handover parameters, i.e. Hyst and TTT, on the basis of certain handover performance indicators (HPI), that reflect the current handover performance of the network. The observed HPIs are: the radio link failures (RLF), detected as failures of the radio link due too low SINR conditions; the handover failures (HOF), detected as too low SINR conditions during the handover execution; the ping-pong handovers, detected as handovers returning to the originating cell within a short time. The HPIs are defined as ratios of counted events divided by the number of handovers for the handover failure ratio $\left(H P I_{H O F}\right)$ and ping-pong handover ratio $\left(H P I_{P P}\right)$ and as ratio of counted events divided by the number of active calls for the radio link failure ratio $\left(H P I_{R L F}\right)$. The HPO algorithm observes the individual HPIs per cell and computes a weighted sum HP according to equation 2 in order to evaluate the current handover performance. The weights have been set to $w_{1}=1$, $w_{2}=0.5, w_{3}=2$, giving the RLF the highest priority.

$$
H P=w_{1} H P I_{H O F}+w_{2} H P I_{P P}+w_{3} H P I_{R L F}
$$

The HPO algorithm chooses the handover operating point (HOP) -so pairs of discrete Hyst and TTT values- out of a defined set, by comparing the HP of the current HOP to that of the previous HOP and hence changes the optimization direction if necessary. The optimization is carried out independently in all cells and aims to achieve reasonable handover performance.

\section{Simulation SCEnARio}

The investigations on the interaction between the handover optimisation and the load balancing algorithms require a

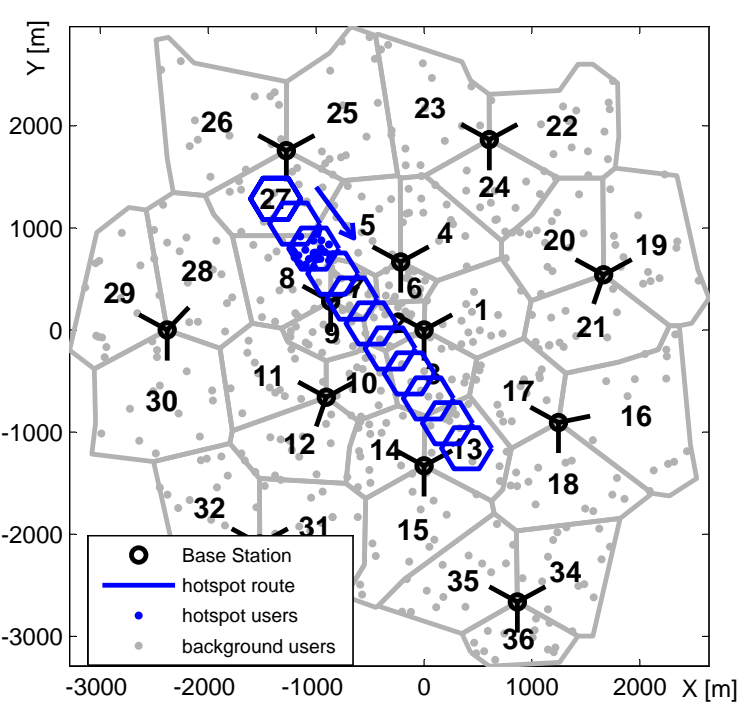

Fig. 2. Simulation Scenario, network layout and indication of the hotspot area

simulation scenario that provides load fluctuations to enable load balancing gains on the one hand and realistic cell shapes to enable handover performance gains using different handover parameters on the other hand. Thus a non-regular network layout [7] has been selected as simulation scenario as shown in Figure 2. All other simulation parameters (radio parameters) are referenced from standard simulation assumptions [4]. The load variations are introduced to this scenario by a moving hotspot, i.e. a group that contains a high number of users concentrated in small area, which moves across the network on a predefined path.

The hotspot starts in cell \#27 and moves to cell \#13. The path is indicated in Figure 2. The number of background users has been set to 14 users per cell and the number of users in the hotspot to 50 users for the present simulations. All users move -after been dropped into the network- with a random waypoint model and a speed of $30 \mathrm{~km} / \mathrm{h}$. The simulation length has been choosen to 20 minutes (realtime). Each of the two algorithms works with a certain optimization interval: 1s for LB and 60 s for the HPO.

\section{INTERACTIONS}

The two SON algorithms described in the previous section may not control exactly the same parameters but they both influence the handover decisions as the parameters cannot be decoupled (see equation 1). Therefore, in this section, potential interactions between LB and HPO algorithms are analyzed and observed in simulation results.

Figure 3 shows an example with an interaction of LB and settings influenced by the HPO. Due to the overload at the SeNB side, the LB function adjusts the handover offsets to adjacent TeNB and thus shifts the virtual border and switching point from point 1 to 2 . In this situation, some users will be outside the SeNB serving area and will be forced to start a handover procedure to the TeNB. Due to the HPI values (LB 


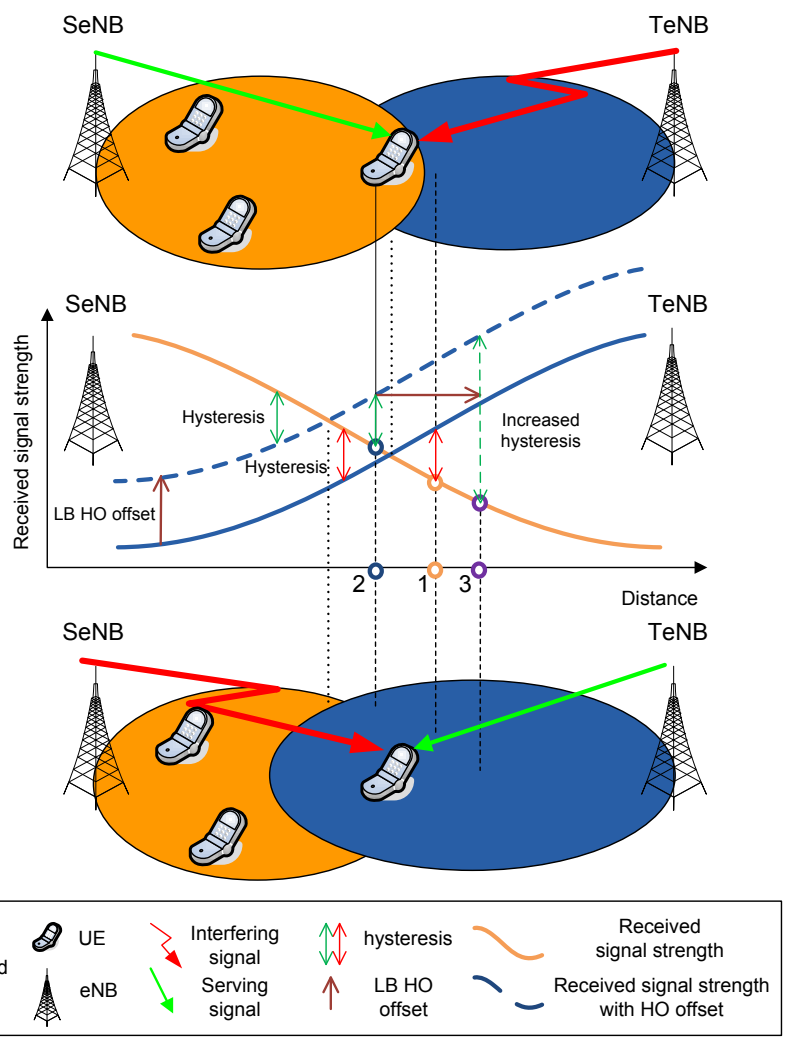

Fig. 3. Load Balancing and Handover Parameters Optimisation interaction

action might introduces additional handovers and thus may increase the monitored HPIs), the HPO algorithm in one of the cells involved in LB optimisation process may decide to increase the hysteresis. A higher hysteresis shifts the switching point to point 3 , which is in contradiction to the aim of the LB optimisation. If the users that were previously handed over during the LB process also participated in a ping-pong handover after the hysteresis was updated to a higher value, they may get stuck again in the cell that was overloaded. The two SON algorithms change different control parameters but reduce the desired effects of the other function. Actions of one function may also intensify the effects of the second function, like in the situation when HPO algorithm allows some users to handover in the direction that is desired the LB algorithm as well by reducing the hysteresis.

Figure 4 shows the HPIs and hysteresis values over time in cell \#6 with both HPO and LB algorithms enabled and Figure 5 shows the number of users served by this cell under the same conditions. Cell \#6 is located very close to the path of the moving hotspot and actively participates in the LB process and is considered as a TeNB for neighbouring overloaded cells (see Figure 5 higher number of users served by cell \#6 in case with LB is switch on). In the simulation without LB (red curve), HPO is triggered by the handover ping-pong ratio (approximately 370s time step) and then after 2 HPO periods, the ping-pongs are reduced to 0 . In the case of the simulation with HPO and LB (blue curve), LB introduce RLFs to the cell and triggers the HPO algorithm much earlier; only this time the HPO cannot manage the situation and different hysteresis adjustments are observed until the end of simulation even after the hotspot has left this cell area.
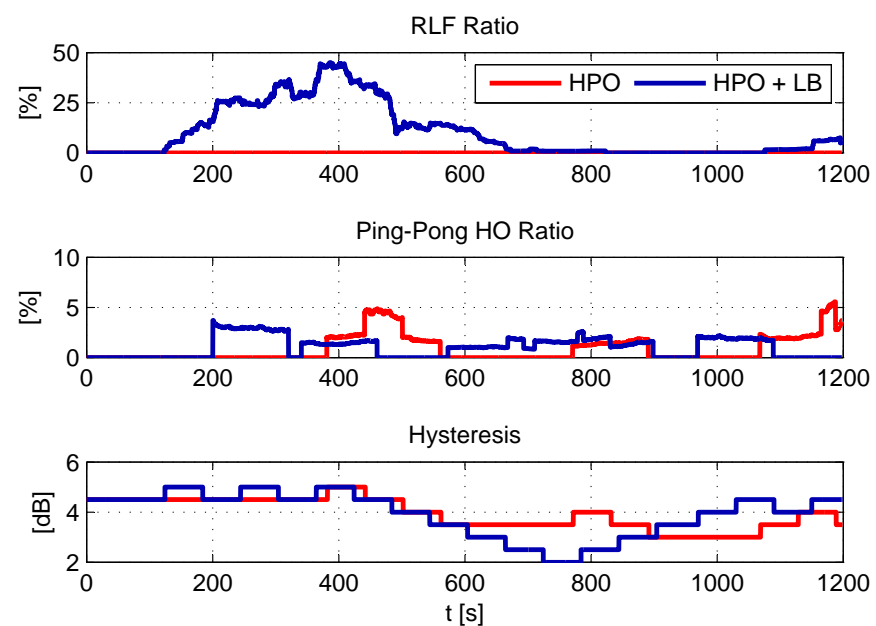

Fig. 4. Load Balancing impact on Hysteresis optimisation; cell \#6

If we assume, that settings adjusted by the HPO algorithm working as a standalone function are optimal, the differences in the hysteresis chart caused by the LB algorithm lead up to the HPIs RLF and ping-pong degradation. The LB algorithm ran in this cell only for a certain time period but worse performance is observed overall simulation not only in the time period when hotspot was moving through cell \#6.

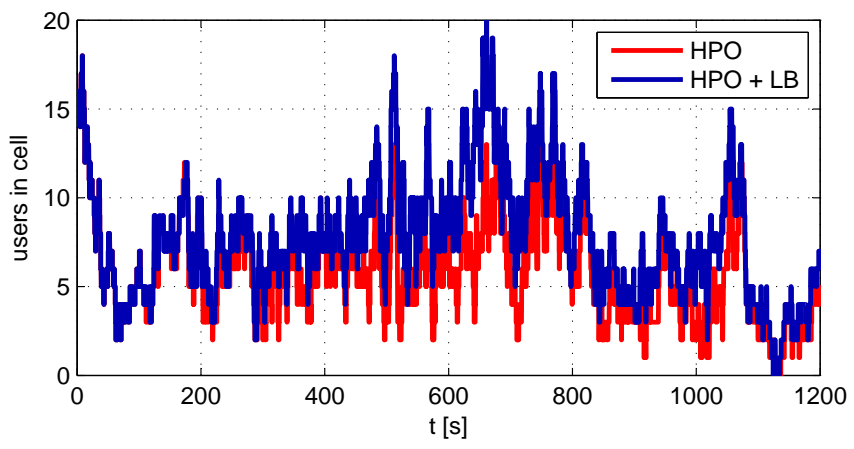

Fig. 5. Number of users served in cell \#6

\section{COORDINATION}

\section{A. Coordination mechanism}

In a real network, the two SON algorithms described here will be active in parallel. As shown in the previous section, there is a strong interaction between the algorithms since LB relies on changing handover parameters to level the load amongst neighboring cells in overloaded network areas. A straightforward solution for integrating both algorithms could be to combine them into one optimization function. The advantage would be that the interest conflicts of the two competing algorithms could be handled within the function. Hence, it 
would not be necessary to define interfaces between the SON algorithms. This can be a viable solution for some combinations of SON algorithms, but it may be hard to extended this approach to a setting with many SON algorithms, as the complexity considerably increases. Beyond that it would be much harder to enhance a single SON algorithm if integrated in one large SON function. This is why we have opted for a coordinator framework instead. A more detailed view on the SON coordinator framework can be found in [9].

The main purpose of the coordinator mechanism is to ensure that the SON algorithms jointly work in the network and still achieve considerable gain in system performance. This requires the avoidance and/or resolution of conflicts between the algorithms. In this section we are proposing a coordination mechanism that evaluates and limits the optimization actions of the SON algorithms if necessary.

The KPI metrics are the basis for the system performance analysis and are used for the assessment of the SON algorithms as well. Hence the KPI statistics are reported to the SON algorithms to analyze the system performance. Based on this analysis the SON algorithms adapt their control parameters if necessary and assess the influence of the parameter changes using the shown and additional KPI metrics, like the satisfied user ratio for example. This functionality has been developed for different SON algorithms as stand-alone solution. The coordinator block that is shown in Figure 6 is a new enhancement that allows for coordination between the SON algorithms. The coordinator tracks control parameter changes as indicated in the schematic overview and aligns the KPI statistics to ensure that all SON algorithms operate on the same statistics. Based on the observed parameter changes and KPI statistics the coordinator limits the operation space of the SON algorithms if necessary.

For the coordination of the HPO and LB algorithms, three coordination functionalities have been defined (COO1, $\mathrm{COO} 2$ and $\mathrm{COO} 3$ ) that are combined in the coordinator block. The coordination functionalities, that will be further described below, can be used one at a time or in combination with the other coordination functionalities.

The COO1 coordination functionality acquires information about currently adjusted handover offsets in the network and evaluates the HPI statistics. In the case the HPI statistics show significant performance degradation in comparison to the last observation period in a cell, the $\mathrm{COO} 1$ reduces the handover offsets of this cell or even switches the LB algorithm off for a time period. The duration of the applied changes (e.g. $10 \%$ of the HPO observation window time), the range of the handover offset changes (e.g. step of $2 \mathrm{~dB}$ ) as well as thresholds to trigger the $\mathrm{COO} 1$ functionality (e.g. HPI $\geq 10 \%$ ) can be adjusted manually or tuned automatically.

The goal $\mathrm{COO} 2$ is action harmonization between the HPO and LB algorithms, i.e. the HPO algorithm is not allowed to counteract the LB algorithm by changing the hysteresis parameter. For example, if a cell is overloaded the LB algorithm is activated and the handover offsets to neighboring cells will be adapted to level the users in the overloaded area.

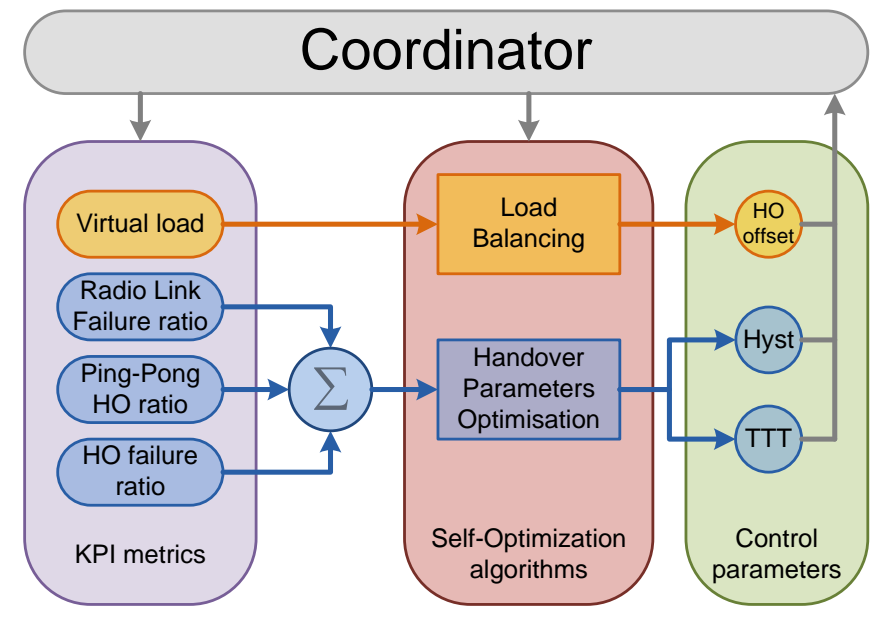

Fig. 6. Coordinator scheme for HPO and LB algorithms

In this case the $\mathrm{COO} 2$ will not allow the HPO algorithm to increase the hysteresis in the overloaded cell since this would keep the users in the overloaded cell which goes against the LB optimization aim. Furthermore the HPO algorithms in the target cells for load balancing handovers will not be allowed to lower the hysteresis since this would increase the number of handovers to the overloaded cell.The $\mathrm{COO} 2$ functionality is triggered during the HPO process if the LB function was active longer than a specified percentage of the previous HPO observation period e.g. $10 \%$.

$\mathrm{COO} 3$ monitors the KPI statistics and detects significant KPI increases (unusual behavior) by comparing the shortterm changes of the KPI to the long-term average. Because of the time relations between the LB and HPO algorithms, the function which applied jeopardy settings is mostly likely to be the LB algorithm (due to more frequent -every secondand drastic changes of the handover offsets -0 to $10 \mathrm{~dB}-$ ). In the case an unusual behavior has been detected the $\mathrm{COO} 3$ functionality cancels all handover offset changes and switches off the LB algorithm for the next few SON periods.

\section{B. Simulation Results}

Results presented in Table I give an overview on the impact of the coordination mechanism used during simulation on observed KPIs and Figure 7 is a graphical presentation of the handover performance HP (equation 2) of the results from Table I. In the reference case, both the HPO and LB algorithms are disabled. The HPO algorithm reduces the number of radio link and handover failures significantly by the cost of a higher ping-pong handover ratio which is a desired effect according to the weighting parameters settings (see section III). The LB algorithm as a standalone function reduces the number of unsatisfied users by more than $75 \%$ but increases the HP metric roughly by $90 \%$. In the case that both optimisation algorithms are activated the number of unsatisfied users is even reduced by almost $80 \%$. However, the HP reach about $15 \%$ higher value than in the reference case without any optimisation. Result obtained from the simulation 
TABLE I

OVERALL RESULTS

\begin{tabular}{c|c|c|c|c}
\hline $\begin{array}{c}\text { SON } \\
\text { algorithm }\end{array}$ & $\begin{array}{c}\text { Unsatisfied } \\
\text { users }\end{array}$ & $\begin{array}{c}\text { HO ping-pong } \\
\text { ratio }\end{array}$ & $\begin{array}{c}\text { HO failure } \\
\text { ratio }\end{array}$ & $\begin{array}{c}\text { Radio Link Failure } \\
\text { ratio }\end{array}$ \\
\hline \hline reference & 8.2 & 0.5 & 1.2 & 8.9 \\
HPO & 7.9 & 1.8 & 0.6 & 5.6 \\
LB & 1.8 & 3.1 & 2.4 & 16.7 \\
HPO+LB & 1.6 & 3.5 & 10 & 9.6 \\
COO & 3.2 & 4.7 & 1.0 & 6.0
\end{tabular}

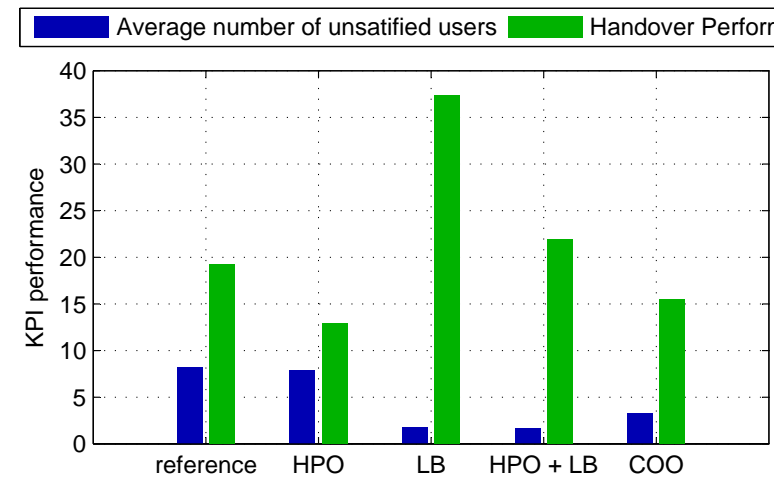

Fig. 7. Average HPO and Load Balancing KPIs over the 20 minutes simulation length with coordination mechanisms
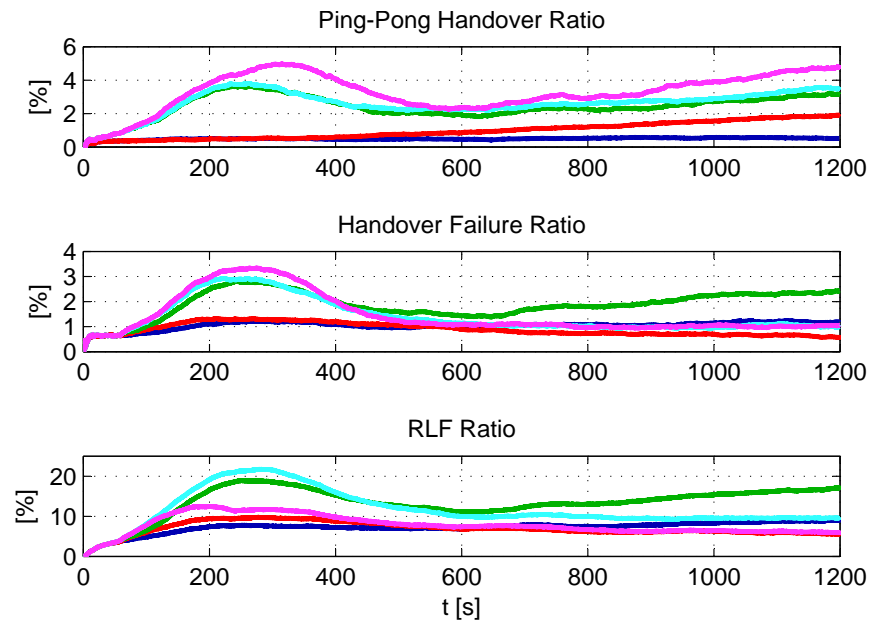

Fig. 8. HPO KPIs, over the 20 minutes simulation length

with coordination mechanism $\mathrm{COO}$ shows a reduction of RLFs occurrence, handover failure ratio and number of unsatisfied users but at the expense of higher handover ping-pong ratio.

Figure 8 shows the timelines for the HPIs and Figure 9 shows number of unsatisfied users for a whole simulation runtime. Algorithms performance can vary over time especially at the beginning of simulations. It is well visible in case of simulation with switched on only LB function (green curve) where KPIs performances are related with the hotspot position in the simulated network. Simulations results obtained from other simulation (apart from Ping-Pong Handover Ratio) after convergence approximately after 400 s and in case of switched on $\mathrm{COO}$ mechanism achieve performance of the standalone LB and HPO case.

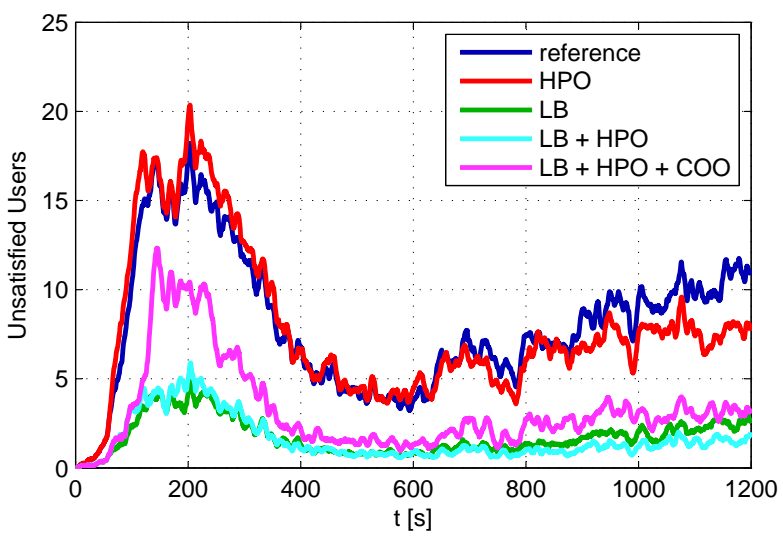

Fig. 9. Unsatisfied users over the 20 minutes simulation length

\section{CONCLUSION AND OUTLOOK}

In this document we present the results of the investigations on the interactions between a handover optimization and load balancing SON algorithms when running in parallel in a cellular mobile network. Although both optimisation algorithms influence the handover decision of the users and hence interact with each other, it is possible to coordinate the load balancing and handover parameter optimization SON algorithms. The coordination algorithm is able to control the algorithms to reasonable system performance or even combining the strengths of both algorithms to a performance better than a single algorithm gain. Note, that the singular algorithms are unchanged, the coordinator takes control on top of the algorithms. This work on coordination systems is extended within the Socrates project with an abstract coordination system steering also other SON algorithms.

\section{REFERENCES}

[1] SOCRATES, Self-optimisation and self-configuration in wireless networks, European Research Project, http://www.fp7-socrates.eu.

[2] A. Lobinger, S. Stefanski, T. Jansen and I. Balan, Load Balancing in Downlink LTE Self-Optimizing Networks, VTC 2010 Spring, Taipei, Taiwan, May, 2010.

[3] I. Viering, M. Döttling, A. Lobinger, A mathematical perspective of self-optimizing wireless networks, IEEE International Conference on Communications 2009 (ICC), Dresden, Germany, May 2009.

[4] 3GPP, Physical Layer Aspects for evolved Universal Terrestrial Radio Access (E-UTRAN), Technical Report TR 25.814, available at http://www.3gpp.org.

[5] T. Jansen, I. Balan, I. Moerman, T.Kürner, Handover parameter optimization in LTE self-organizing networks, COST $2100 \mathrm{TD}(10) 068$, Joint Workshop COST 2100 SWG 3.1 \& FP7-ICT-SOCRATES, Athens, Greece, February 5, 2010.

[6] 3GPP, Evolved Universal Terrestrial Radio Access Network (E-UTRAN); X2 Application Protocol (X2AP), Technical Specification TS 36.423, available at http://www.3gpp.org.

[7] J. Turkka and A. Lobinger, Non-regular Layout for Cellular Network System Simulations, PIMRC 2010, Istambul, Turkey, September, 2010.

[8] 3GPP TS 36.331 V9.2.0 (2010-03), Evolved universal Terrestrial Radio Access (E-UTRA); Radio resource control (RRC); Protocol specification (Release 9), March 2010

[9] M. Amirijoo, A. Eisenblaetter, R. Litjens, M. Neuland4, L.C Schmelz, J. Turk, A Coordination Framework for Self-Organisation in LTE Networks, 12th IFIP/IEEE International Symposium on Integrated Network Management (IM'2011)to be held 23-27 May 2011 